\title{
Discriminant Method of Sand Liquefaction
}

\author{
Xiaodong Peng ${ }^{1,2^{*}}$ \\ ${ }^{1}$ College of Civil Engineering and Architecture, Shandong University of Science and Technology, Qingdao, Shandong 266590, China \\ ${ }^{2}$ Key Laboratory of Civil Engineering Disaster Prevention and Mitigation, Shandong University of Science and Technology, Qingdao, \\ Shandong 266590, China;
}

\begin{abstract}
With the proposal of 1.8 billion mus of cultivated land red line and the rise of land reclamation in China, more and more national defense and civil projects are built on poor geological soil, such as reclamation reefs in the South China Sea. The liquefaction damage caused by earthquake, such as water spraying, grouting and surface cracks, has a great impact on such buildings, and poses a threat to people's lives and property. Therefore, it is of great significance to study the liquefaction of sand in foundation soil. Since Academician Huang Wen-xi first proposed to use indoor dynamic triaxial test to study liquefaction, a series of achievements have been made in the liquefaction of foundation soil. The research methods of sand liquefaction have developed from simple single sample of numerical simulation, numerical simulation and test. Referring to the literature at home and abroad, this paper sorted out the discriminant method of sand liquefaction, experimental study of sand liquefaction and post-liquefaction analysis.
\end{abstract}

\section{Introduction}

With the rapid development of economy and the increasing frequency of human activities, it has brought some great influence to the activities inside the earth. In recent years, some strong destructive earthquakes have occurred all over the world, such as the 8.3 earthquake in San Francisco, USA on April 18, 1906, the 7.9 earthquake in Kanto, Japan on September 1, 1923, the 7.1 earthquake in Fukui, Japan on June 28, 1948, and the 8.0 earthquake in Wenchuan, China on May 12, 2008. After the earthquake, it has brought great damages and inconvenience to people's production and life, resulting in great economic losses and casualties. According to incomplete statistics, the high death rates after the earthquake is mainly caused by the collapse of buildings. For the collapse of buildings, there are many reasons, such as the building seismic grade is not high, the building material strength grade is not high, or the building foundation soil liquefaction occurs under the action of earthquake force, resulting in the damage of buildings. With the continuous development of modern science and technology and the application of computer big data, there is a technical support for the occurrence and prediction of earthquakes. Therefore, it is also of great significance of the seismic research of buildings, especially for the problem of foundation soil.

Huang Wenxi is a creative scholar in the study of sand liquefaction mechanism in China [1]. As early as the late 1960s, Huang Wenxi proposed that the dynamic triaxial apparatus should be used to study the liquefaction of soil. Wang Yajun [2] proposed the pore pressure failure model of marine sand in Zhoushan sea area. The appearance of sand samples was analyzed by XRD and SEM to study the influence of appearance shape of Marine Soil on soil liquefaction, and the pore pressure model of liquefaction failure of sand under cyclic excitation was established. Liu Hanlong [3] and others have carried out a series of experimental studies on calcareous sand by using dynamic triaxial apparatus, analyzed the mechanical characteristics of liquefaction of calcareous sand in the South China Sea, and seed, Finn $[4,5]$ and others have carried out a large number of experiments since the 1960s, and put forward the widely used simple and convenient method, which has made outstanding contributions to the study of sand liquefaction in the field of geotechnical engineering.

\section{Liquefaction judgment}

At present, there are many methods to distinguish sand liquefaction at home and abroad, which can be divided into traditional methods and non-traditional methods according to different ways. Although the two methods are different, they have their own advantages and disadvantages, which is mainly due to the complexity of factors affecting sand liquefaction.

\subsection{Traditional discrimination method}

The traditional discrimination method can be divided into field tests and indoor test.

The discrimination mechanism of field test is: in the macro seismic liquefaction and non liquefaction areas, according to the data of discrimination index measured by field test, through analysis, statistics and summary, the relationship between the data and macro seismic disaster

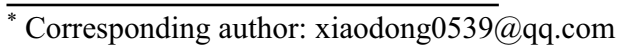


data is established, and the empirical formula or liquefaction boundary is obtained to distinguish liquefaction or not.

(1) Criterion of critical blow count of standard penetration(SPT)

$$
\mathrm{N}<\mathrm{N}_{\text {cr }}
$$

Where, $\mathrm{N}$ is the actual number of SPT blows; $\mathrm{N}_{\mathrm{cr}}$ is the critical number of SPT for liquefaction.

According to code for seismic design of buildings (GB 50011-2010), the critical number of SPT for liquefaction is calculated as follows:

$$
\mathrm{N}_{\mathrm{cr}}=N_{0} \beta\left[\ln \left(0.6 d_{s}+1.5\right)-0.1 d_{w}\right] \sqrt{3 \% / \rho_{c}}
$$

Where, $\mathrm{N}_{\mathrm{cr}}$ is the critical value of SPT blow count for liquefaction identification; $N_{0}$ is the benchmark value of SPT blow count for liquefaction identification; $d_{\mathrm{s}}$ is the SPT depth of saturated soil $(\mathrm{m}) ; d_{\mathrm{w}}$ is the groundwater level $(\mathrm{m}) ; \rho_{\mathrm{c}}$ is the percentage of clay content, which should be $3 \%$ when it is less than $3 \%$ or sandy soil; and $\beta$ is the adjustment coefficient, which is 0.80 for the first group, 0.95 for the second group and 1.05 for the third group.

According to the code for geological exploration of water resources and Hydropower Engineering (GB 504872008), the following formula is used to calculate the critical standard number of penetration for liquefaction:

$$
\mathrm{N}_{\mathrm{cr}}=N_{0}\left[0.9+0.1\left(d_{s}-d_{w}\right)\right] \sqrt{3 \% / \rho_{c}}
$$

Where, $\rho_{\mathrm{c}}$ is the percentage of clay content, $3 \%$ should be used when it is less than $3 \% ; N_{0}$ is the benchmark value of standard penetration blow count for liquefaction identification; when the standard penetration point is within $5 \mathrm{~m}$ below the ground, $5 \mathrm{~m}$ should be used for calculation.

According to the formula proposed in code for seismic design of buildings (GB 50011-2001), the formula is as follows:

$$
\left.\mathrm{N}_{\mathrm{cr}}=N_{0}\left[2.4-0.1 d_{w}\right)\right] \sqrt{3 \% / \rho_{c}}
$$

For liquefiable soil layer, the liquefaction grade is determined according to the liquefaction index IIE calculated by the following formula:

$$
I_{i e}=\sum_{i=1}^{n}\left(1-\frac{N_{i}}{N_{c r i}}\right) d_{i} w_{i}
$$

Where: $I_{\mathrm{ie}}$ - Liquefaction index; $N_{\mathrm{i}^{-}}$Measured SPT blow counts of point I in saturated soil; $N_{\text {cri- }}$ Critical SPT blow count corresponding to $N_{\mathrm{i}}$ depth; $N$ - total number of SPPs in saturated soil layer within $15 \mathrm{~m}$ depth of each borehole.

The classification is as follows:

Table1. Classification standard of sand liquefaction grade

\begin{tabular}{|l|c|c|c|}
\hline Liquefaction grade & slight & secondary & serious \\
\hline $\begin{array}{l}\text { Liquefaction index } \\
\text { at 15m depth }\end{array}$ & $0<I_{\mathrm{LE}}<5$ & $5<I_{\mathrm{LE}}<15$ & $I_{\mathrm{LE}}>15$ \\
\hline $\begin{array}{l}\text { Liquefaction index } \\
\text { at 20m depth }\end{array}$ & $0<I_{\mathrm{LE}}<6$ & $6<I_{\mathrm{LE}}<18$ & $I_{\mathrm{LE}>18}$ \\
\hline
\end{tabular}

(2) Cone penetration test (CPT)

According to the code for investigation of Geotechnical Engineering (2001), the discrimination method based on CPT test data is an empirical formula based on the measured seismic data. The method considers the different effects of near and far earthquakes, and the influence of clay content on liquefaction

$$
\begin{gathered}
p_{s c r}=p_{s 0} \alpha_{w} \alpha_{u} \alpha_{p} \\
q_{c c r}=q_{c 0} \alpha_{w} \alpha_{u} \alpha_{p} \\
\alpha_{w}=1-0.065\left(d_{w}-2\right) \\
\alpha_{u}=1-0.050\left(d_{u}-2\right)
\end{gathered}
$$

Where: $p_{\text {scr }}, q_{\text {ccr }^{-}}$Critical values of CPT specific penetration resistance and cone resistance of saturated soil (MPA); $d_{\mathrm{w}}$-Groundwater depth; $d_{\mathrm{u}}$-Thickness of overly non liquefied soil layer; $\alpha_{\mathrm{w}}$-the correction factor of groundwater depth, $\alpha_{\mathrm{w}}=1.13 ; \alpha_{\mathrm{u}}$-Correction factor of overly non liquefiable soil thickness.

When the measured specific penetration resistance or cone tips resistance is less than the critical value of penetration resistance or cone tip resistance of penetration liquefaction, it should be judged as liquefied soil, otherwise the sand will not be liquefied.

For the field test method, there are the following methods: shear to wave velocity method, Rayleigh wave velocity method and energy discrimination method. For the above methods, the phenomenon of sand liquefaction can be seen intuitively, and multiple factors affecting sand liquefaction can be considered, which can avoid the disturbance of sample preparation for laboratory tests, and has certain practicability and reliability, but there are also some shortcomings: 1 , The accumulated field test data of all kinds of soil liquefaction are relatively few; 2 . The investigation data onto foundation liquefaction are mostly obtained in free site, generally speaking, this kind of method is suitable for liquefaction identification of free site; 3. This kind of method is based on liquefaction examples of earthquake site, which is regional and not universal.

Laboratory tests methods mainly include dynamic triaxial test, centrifuge test and shaking table test.

(1) Dynamic triaxial test

As early as the late 1960s, Huang Wenxi proposed that the dynamic triaxial apparatus should be used to study the liquefaction of soil. Since then, China has opened the door to the study of sand liquefaction by using dynamic triaxial apparatus, and a series of achievements have been made. The dynamic triaxial apparatus is shown in Figure 1.

In 2015, Liu Hanlong et al. Studied the dynamic liquefaction characteristics of calcareous sand in the South China Sea by using geotechnical dynamic triaxial apparatus, and analyzed the dynamic stress-strain relationship and dynamic pore pressure characteristics of calcareous sand through experiments. The results show that: (1) under the condition of isobaric consolidation, the deformation mode of saturated calcareous sand under different confining pressures is the same, and the cumulative plastic strain increases with the increase of vibration times. (2) Under the condition of isobaric consolidation, the ability of resisting deformation is stronger at the initial stage of loading, and the strain amplitude increases to the increase in loading times. 


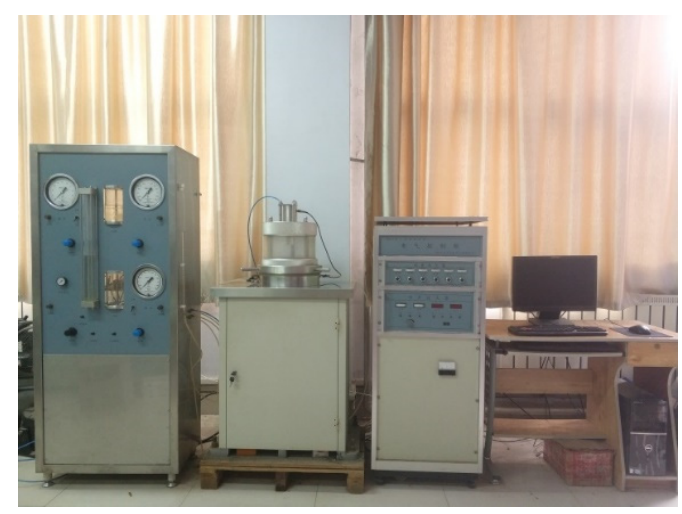

Fig.1. Dynamic triaxial instrument

(2) Shaking table test

In 2005, Meng Shangjiu et al studied the mechanism of non-uniform seismic settlement of buildings on liquefiable foundation of shaking table tests. The shaking table test of liquefiable soil structure interaction was designed and realized. The test can be divided into two types, one is large shaking table test with seismic wave input, the other is small shaking table test of sine wave input. The input of waveform is mainly the time history of unidirectional horizontal acceleration, and the dynamic stress of symmetrical basements, pore pressure and uneven seismic subsidence of symmetrical apex of building are obtained.

In this test, the sand box is used to simulate the foundation. The sample sizes of the large and small shaking table are $160 \mathrm{~cm} \times 90 \mathrm{~cm} \times 120 \mathrm{~cm}$ and $51 \mathrm{~cm} \times$ $34 \mathrm{~cm} \times 32 \mathrm{~cm}$ respectively. The gray fine sand is used as the foundation soil, and the water sinking method is used to form the foundation. The experimental results show that (1) due to the difference between pore pressure on both sides with the symmetrical structure, the building will topple in the direction where the pore pressure is leading. (2) One of the decisive factors affecting the increase of pore water pressure is the vertical dynamic stress of the base.

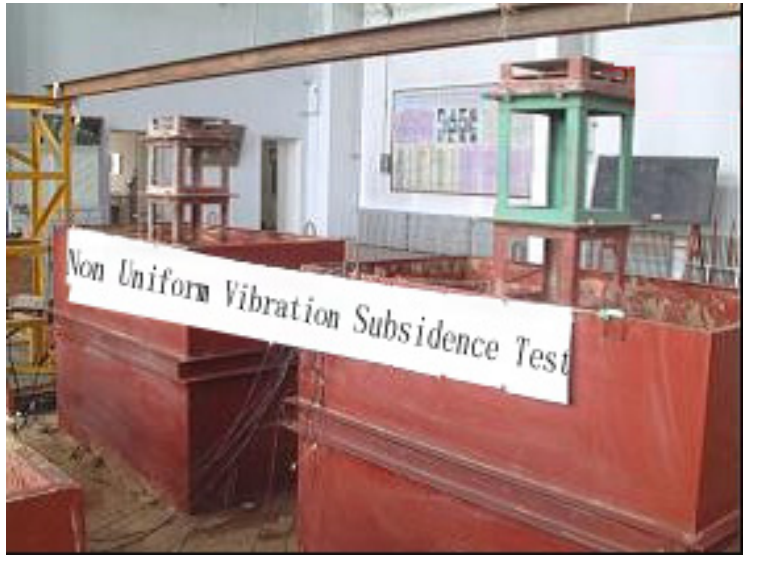

Fig.2. Shaking table test

(3) Centrifuge tests

The 1990s is a new stage of shaking table test research on pile-soil-structure dynamic interaction. Shaking table test of pile-soil dynamic interaction under sandy soil liquefaction is a hot spot of this stage. A large number of pile foundation failures of Loma Prieta earthquake and Kobe earthquake further promote the experimental research on dynamic interaction, It not only pays attention to the study of the similarity ratio of the test device and model, but also more and more compare the test results from the theoretical analysis results to test the rationality of the selection of soil parameters in the theoretical analysis. In this stage, the United States and Japan started the edus joint research project, and carried out a large number of shaking table tests. Before 1995, small-scale tests were mainly carried out. After 1995, a large number of large-scale and even prototype tests were carried out in Japan on a large-scale shaking table with a capacity of $15 \mathrm{~m} \times 15 \mathrm{~m}$ and a load of 500t. Edus research results have been published one after another, and the important enlightenment to the research work is: (1) model shaking table test can get very consistent results with centrifuge test, so we should vigorously develop economic smallscale model shaking table test and improve centrifuge test technology level; (2) numerical simulation and theoretical research of pile soil structure under site liquefaction condition, The global analysis method based on effective stress and the nonlinear Winkler method. The assumed parallel spring damper analysis method of foundation beamed has been developed, and the results are consistent with the experimental results, which are also the direction of further development in the future.

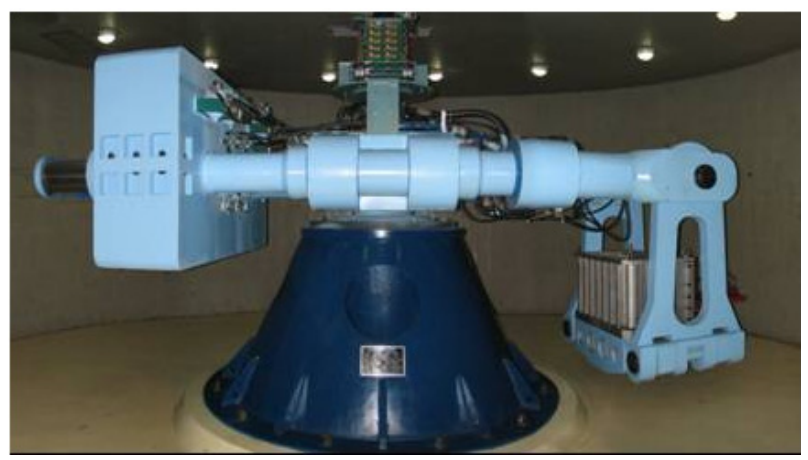

Fig.3. Centrifuge test

\subsection{Unconventional discrimination method}

Unconventional discrimination method includes neural network method and fuzzy comprehensive evaluation method. The following neural network method is introduced.

(1) Artificial neural network method

Artificial neural network (ANN) is a new discriminant method, which mainly combines the global search ability of genetic algorithm with the guiding ideology of BP algorithm. It is based on the application of big data by using MATLAB. In 2012, Fan Fu-song and others used the generalized regression neural network to analyze the discrimination method of sand liquefaction. Based on a large number of experimental data onto a certain place, the standard method and seed method were used to judge the liquefaction respectively, and then the data onto the same discrimination results were selected as samples, and the generalized regression neural network was used to discriminate it twice. 


\section{Conclusion}

(1) Although there are many researches on sand liquefaction, the research on sand liquefaction is still an important research content of the field of geotechnical engineering, and the test method can be further improved.

(2) Using special numerical simulation software can simulate the problem of sand liquefaction. Comparing the numerical simulation results from the experimental results, we can draw the corresponding conclusions. Therefore, the numerical simulation method to study the problem of sand liquefaction may become a new trend in the future.

(3) With the continuous maturity of big data technology, the non-traditional methods of sand liquefaction research will become more and more mature, which plays an important role in the auxiliary test verification of sand liquefaction.

\section{References}

1. W Z Wang. Experimental study on vibration pore water pressure of saturated sand[J]. Journal of water conservancy, 1962(02): 37-47.

2. Y J Wang, F Jin, C H Zhang, et al. Liquefaction failure pore-pressure models of zhoushan ,arine sandy soil under cyclic exciting loading $[\mathrm{J}]$. Chinese Journal of Rock Mechanics and Engineering, 2013, 32(03): 582597.

3. H L Liu, D Hu, Y Xiao, et al. Test Study on Dynamic Liquefaction Characteristics of Calcareous Sand[J]. Journal of Disaster Prevention and Mitigation Engineering, 2015, 35(06): 707-711+725.

4. Finn W D L, Lee K W, Martin G R.An effective stress model for liquefaction.Jarnal of the Geotechnical Engineering Division, ASCE, 1997, 103(6): 517-533.

5. Seed H B, Idriss I M. A simplified procedure for evaluation soil liquefaction potential.J of Geot Eng Dw, ASCE, 1971, 97(2): 201-255. 